\title{
Multi-objective Optimization of Combined Cooling, Heating and Power System
}

\author{
Guanwu Lin*, Xiaolan Wang \\ College of Electrical and Information Engineering, Lanzhou University of Technology, Lanzhou 730050, China
}

Corresponding Author Email: only_uu@ 126.com

https://doi.org/10.18280/ejee.210202

Received: 18 January 2019

Accepted: 12 March 2019

\section{Keywords: \\ multi-objective optimization, combined cooling, heating and power (CCHP) system, artificial bee colony $(A B C)$ algorithm}

\begin{abstract}
This paper designs a combined cooling heating and power (CCHP) system, which realizes cooling function with an absorption refrigerator and an electric refrigerator. The main devices of the system were analyzed and modelled separately. Next, a multi-objective optimization model was established to improve the system performance in terms of energy efficiency, operation cost and greenness. Meanwhile, the population coding and selection process of multi-objective artificial bee colony (ABC) algorithm was improved to solve the proposed optimization model. Finally, the proposed optimization model was verified through simulation experiments. The research findings shed new light on the operation features of the CCHP system under the optimization strategy, and the coupling between the different objectives.
\end{abstract}

\section{INTRODUCTION}

Combined cooling, heating and power (CCHP) system [1] makes cascade utilization of energy, and generates electricity, and achieves refrigeration, heating and power generation at the same time. In traditional energy utilization systems, the power generation efficiency is merely $40 \%$, that is, the majority (60\%) of energy is wasted. The CCHP system pushes up the energy utilization rate to $80 \%$ by recovering the waste heat from power generators. Another advantage of the system lies in the reduced emission of $\mathrm{CO}_{2}$ and other pollutants.

The CCHP system, featured by the coupling among multiple energies, encompasses devices of various technical categories and structural forms [2-3]. The system devices include power generators, refrigerators, heaters, energy storage devices and waste heat recovery systems. There are many kinds of CCHP systems, that vary in terms of energy type, device model and system scale [4-6].

The existing CCHP systems are often categorized by power generators, for the diversity of system process hinges on the variability of power technology. In fact, power generators capable of thermoelectric conversion are cornerstones of the CCHP system. In the CCHP system, heat-driven refrigerators are the main devices that utilize waste heat. These refrigerators may differ greatly in refrigeration principle, driving media and working efficiency [7]. In addition, there are marked differences between the waste heats from different power plants, and between absorption refrigerators in the temperature and form of driving heat source.

Facing the vast differences above, it is necessary to properly select and configure every device in the CCHP system. Otherwise, the system capacity will fall short of or exceed the requirement, failing to achieve the desired performance. This calls for a rational and effective way to optimize the design of the CCHP system, coordinate the operation between all components, and maximize the system benefits. The design optimization needs to tackle many issues, including but not limited to device selection, capacity allocation and parameter design.
The design optimization is by no means an easy task. The strong coupling in the CCHP system requires careful weighing of energy efficiency, economy, environment and social benefit. To make matters worse, the CCHP system performance will be dampened greatly by off-design operation. In severe cases, the system will fail to save energy and reduce emission, but lead to even greater waste. Therefore, an effective and feasible energy management strategy must be integrated to the design optimization.

In light of the above, this paper designs a CCHP system that realizes the cooling function with an absorption refrigerator and an electric refrigerator. The main devices of the system were analyzed and modelled separately. Next, a multiobjective optimization model was established to improve the system performance in terms of energy efficiency, operation cost and greenness. Meanwhile, the population coding and selection process of multi-objective artificial bee colony (ABC) algorithm was improved to solve the proposed optimization model. Finally, the proposed optimization model was verified through simulation experiments. The research findings shed new light on the operation features of the CCHP system under the optimization strategy, and the coupling between the different objectives.

\section{LITERATURE REVIEW}

The CCHP system is highly nonlinear, with strong coupling between various energies. To improve the system performance, many simulations, computations and experiments have been conducted to derive system models, coupling relationship, optimal design and operation control.

In the field of mechanism modelling, Fang et al. [8] established simulation models of the CCHP system based on various factors, such as variable efficiency, energy consumption of peripheral devices, and number of devices, and explored the impact of each model on operation cost. Taking gas turbine and other devices as black boxes, Arosio et 
al. [9] identified the parameters of CCHP system performance, created a simulation model of the system, and disclosed the effect of each parameter on the performance of small-scale combined power system. Teng et al. [10] set up a mixed integer linear programming model for cogeneration system, and optimized the system performance in terms of investment cost, energy cost and $\mathrm{CO}_{2}$ emission. Liu et al. [11] derived the real-time energy flow function of cooling, heating and power, optimized the dispatch under the traditional operation mode, and improved the economy and greenness of the CCHP system. Using semi-tensor product (STP) operation, Zhang et al. [12] transformed fuzzy reasoning and rule-based system into matrix form, developed a matrix model of the fuzzy relationship between input and output variables, and validated the model through simulation and experiment. Jing et al. [13] modelled the CCHP system by the fuzzy multi-criteria decision-making model.

In the field of design optimization, Ali et al. [14] proposed an optimal allocation model for the CCHP system, and solved it by genetic algorithm (GA) to obtain the optimal capacity and operation mode of the system. Pantaleo et al. [15] introduced heat pump technology into traditional cogeneration system, optimized the system by mixed integer programming, and verified through simulation that the optimized system outperformed separate power system by $10 \%$ in primary energy utilization rate. Jayasekara et al. [16] established a nonlinear optimization model for combined power system, and compared the system performance indices (e.g. cost recovery period and economy) under different energy policies. Jing et al. [1] designed a CCHP system with solar energy, optimized the design of system components like solar energy collector and photovoltaic power generator, and thus improved the system's energy efficiency and greenness. Considering the configuration of hybrid system, Wang et al. [17] set up an optimization model for the maximal primary energy utilization rate and minimal primary energy emission, and contrasted the optimal capacity of devices under different combinations of weight coefficients in each objective function.

To sum up, the existing studies on the design optimization of the CCHP system rarely consider the reliability of main devices under different operation conditions, making it difficult for the optimized system to adapt to actual conditions. What is worse, the constraints between key parameters and system performance have not been fully exploited, such that the optimal designs cannot balance the interests of different shareholders. Therefore, this paper attempts to achieve desired system performance by adjusting device capacity and key parameters in the design process.

\section{DESIGN OPTIMIZATION OF THE CCHP SYSTEM}

Despite the variety of relevant fields, descriptive forms and solutions, the design optimization of the CCHP system can be summed up as the unified mathematical formula below:

$$
\left\{\begin{array}{cl} 
& \min f(x) \\
\text { s.t. } & h_{i}(x)=0 \quad i=1,2, \ldots, p, p<n \\
& g_{i}(x) \leq 0, \quad j=1,2, \ldots, m
\end{array}\right.
$$

where $x$ is a $n$-dimensional variable; $f(x)$ is the objective function; $h(x)$ is the equality constraint on $x ; g(x)$ is the inequality constraint on $x$. Thus, the system optimization aims to obtain a $x$-solution that maximizes or minimizes the value of the objective function under the constraints.

With the aim to improve energy efficiency, economy and greenness, the design optimization of the CCHP system is a typical multi-objective optimization problem. The basic flow of the problem is illustrated in Figure 1 below.

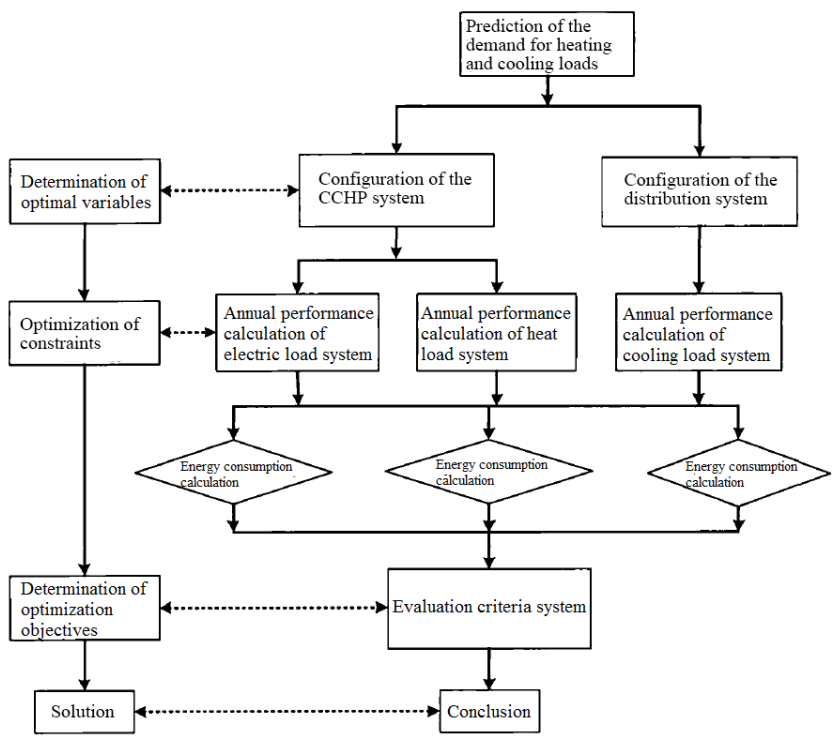

Figure 1. Basic flow of the design optimization of the CCHP system

The CCHP system performance can be improved effectively by adjusting the technical and economic parameters. Nonetheless, it is difficult to change these parameters significantly under the existing conditions. In addition, the parameters differ greatly in their effect on system performance. Therefore, the author decided to enhance the system performance by optimizing the capacity of main units and the key operation parameters.

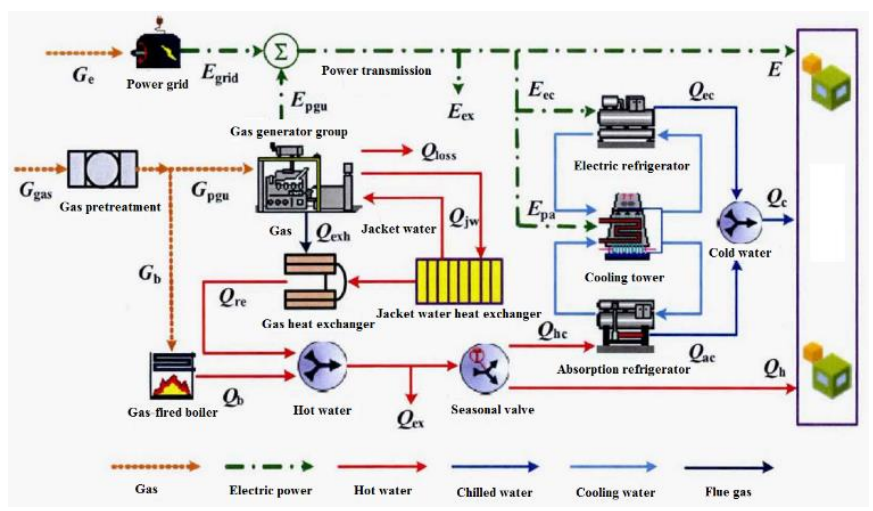

Figure 2. Structure of our CCHP system

The CCHP system can be regarded as the integration between a combined heat and power (CHP) system and the cooling module. The cooling function is realized by the refrigeration technology driven by waste heat. The main devices are mostly absorption refrigerators. However, the amount of waste heat is very limited due to the small size of the power generators. Thus, the system only supports single-effect absorption refrigerators with low coefficient of performance (COP), which exerts a negative effect on system performance in summer. To solve the problem, a 
high-COP electric refrigerator was added to realize mixed cooling. After the modification, the CCHP system is described in Figure 2.

After the addition of the high-COP electric refrigerator, the power supply-consumption balance of our CCHP system at time $t$ can be expressed as:

$$
E_{\text {pgu }}(t)+E_{\text {grid }}(t)=E_{p a}(t)+E_{e c}(t)+E(t)
$$

where $E_{e c}(t)$ is the power consumption of the electric refrigerator at time $t$. The value of $E_{e c}(t)$ can also be computed by:

$$
E_{e c}(t)=\frac{Q_{e c}(t)}{I_{C O P}}
$$

where $Q_{e c}$ is the refrigeration capacity of the electric refrigerator; $I_{C O P}$ is the $\mathrm{COP}$ of the electric refrigerator. Because mixed refrigeration is adopted in summer, the ratio coefficient of electric refrigeration can be defined as: $\beta=$ $\frac{Q_{e c}}{Q_{e c}+Q_{a c}}$

Considering the complex structure and numerous devices in the CCHP system, the design optimization should pay special attention to the selection of specific variables. In fact, most devices in the system are slave peripherals. Their capacities can be determined easily according to the capacity of the active device.

In this paper, the capacity of internal combustion engine $N_{\text {pgu }}$, device start-stop coefficient $\alpha$ and electric refrigeration ratio coefficient $\beta$ are taken as optimization variables. Drawing on the structure of the traditional CCHP system, our CCHP system was optimized to achieve three objectives: energy efficiency, economy and greenness. The three objectives were measured by energy saving rate (ESR), annual cost recovery rate $\left(F_{A C R}\right)$ and $\mathrm{CO}_{2}$ emission reduction rate (ERR), respectively.

In multi-objective optimization problems, it is inevitable for the different objectives to have conflicts. Thus, the optimization result is actually a compromise between the multiple objectives. The weight factors are often introduced to describe the tradeoffs. Different combinations of the weight factors will lead to different optimization results. Therefore, the multi-objective optimization function of our CCHP system can be expressed as:

$$
\max V=\omega_{1} F_{E S R}+\omega_{2} F_{A C R}+\omega_{3} F_{E R R}
$$

where $\omega_{1}, \omega_{2}$ and $\omega_{3}$ are the weight factors of the ESR, $F_{A C R}$ and ERR, respectively; $V$ is the comprehensive optimization objective. The three weight factors must satisfy the following equality constraint of energy balance: $\omega_{1}+\omega_{2}+\omega_{2}=1$

The following inequality constraints were added to ensure the rationality of our model in actual operation:

$$
\begin{gathered}
N_{\min } \leq N_{\text {pgu }} \leq N_{\max }, 0 \leq a \leq \alpha_{\max } \\
0 \leq \beta \leq 1, G_{b} \geq 0, G_{\text {pgu }} \geq 0 \\
0 \leq Q_{a c} \leq Q_{a c_{-} \text {max }}, 0 \leq Q_{e c} \leq Q_{e c_{-} \max } \\
E_{\text {grid }} \geq 0
\end{gathered}
$$

where $N_{\max }$ and $N_{\min }$ are the upper and lower bounds of the capacity of the electric generator; $\alpha_{\max }$ is the upper bound of the minimum load rate of devices. These inequality constraints limit the optimal variables to a reasonable range.

\section{OPTIMAL SOLUTION BASED ON MULTI- OBJECTIVE ABC ALGORITHM}

The basic model of the ABC algorithm [18] mimics the foraging behavior of bees in the search for the optimal solution. Each honey source is viewed as a feasible solution to the target optimization problem, and described as an $n$-dimensional vector. The solutions in the feasible range of the problem form a population, in which each individual corresponds to a honey source. Thus, the population size equals the number of honey sources.

The search starts with the random initialization of the population. At the beginning, a number of honey sources are generated randomly within the feasible range. The number of honey sources is the size of the initial population. The initial positions of the honey sources can be defined as:

$$
x_{i j}=x_{\min , j}+\operatorname{rand}[-1,1] *\left(x_{\max , j}-x_{\min , j}\right)
$$

where $i=1,2, \ldots, N$ is the serial number of honey source; $j=1,2, \ldots, D$ is the dimension of the solution vector; $x_{i j}$ is the $j$ th coordinate component of the $i$-th honey source; $x_{\max , j}$ and $x_{\min , j}$ are the maximum and minimum values of $x_{i j}$ on the $j$ th coordinate component, respectively.

After initialization, the employed bee, the onlooker and the scout look for the honey sources sequentially through the iterative process. The employed bee and the onlooker can search for new honey sources near their original honey sources. The position of each new honey source $x_{i j}^{\prime}$ can be described as:

$$
x_{i j}^{\prime}=x_{i j}+\operatorname{rand}[-1,1] *\left(x_{i j}-x_{k j}\right)
$$

where $k \neq i$ is a random number in $[1, \mathrm{~N}]$. The onlooker identifies the employed bee with the best fitness through roulette selection, and carries out neighborhood search around the identified individual to determine new excellent individuals.

$$
p(\mathrm{i})=\frac{f(i)}{\sum_{j=1}^{N} f(i)}
$$

After a honey source $x_{i}$ has been searched for a limited number of times, the quality of the honey source does not change. In this case, the original bee at the source leaves, and the corresponding employed bee or onlooker will transform into a scout. Then, the scout will randomly select a new honey source through global search and use it to replace the original source. In this way, the algorithm avoids the local optimum trap and converges to the global optimum. In each iteration, the good individuals are retained and bad ones are eliminated. Finally, the global optimal solution can be achieved. The specific flow of the multi-objective $\mathrm{ABC}$ algorithm is presented in Figure 3 below.

In the basic $\mathrm{ABC}$ algorithm, the quality of each solution is evaluated by its fitness, which is computed by the objective function. Considering the conflicting between multiple objectives in the optimization problem, the method to evaluate solution quality should also proceed from the multi-objective perspective. 


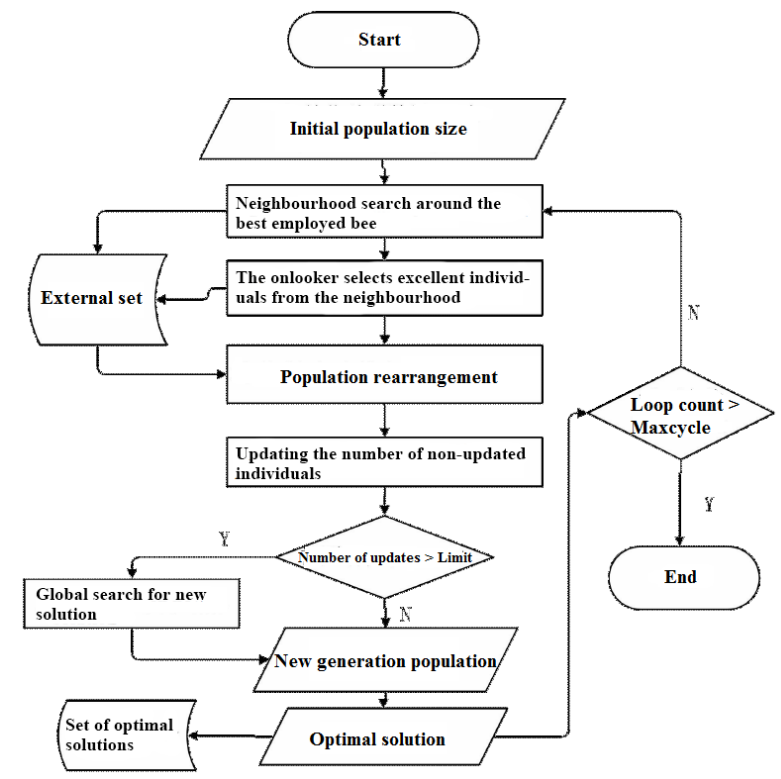

Figure 3. The specific flow of the multi-objective $A B C$ algorithm

According to Pareto dominance model, a solution should rank non-dominantly before individual evaluation. The purpose of sorting is to prepare for the selection and elimination of the later solutions. In practice, the results of non-dominated sorting should be normalized by:

$$
f(i)=\frac{L_{\operatorname{Max}}-L_{i}}{L_{\operatorname{Max}}}
$$

where $L_{i}$ is the Pareto level of individual $i ; L_{\operatorname{Max}}$ is the highest dominance of the current population.

The interaction between local search and global search is the key to the operation of swarm intelligence algorithms like the $\mathrm{ABC}$. To enhance the search ability of the $\mathrm{ABC}$ algorithm, the search formula was improved to make local search results closer to the optimal solution:

$$
x_{i j}^{\prime}=x_{i j}+T * \operatorname{rand}[-1,1] *\left(x_{i j}-x_{i j}^{\prime}\right)
$$

where $T$ is the adaptive adjustment of parameters in neighborhood search formula.

Another focal point of multi-objective optimization is to define the solution structure and assure the new solutions are feasible scheduling solutions. The solution structure is usually expressed as a matrix or a vector [19]. The latter means ranking the solutions by vectors. During decoding, the processing stages are gradually promoted according to heuristic principles.

In this paper, the vector coding is improved into a chromosome coding method. Let solution vector $X_{i}=$ $\left(S_{1}, S_{2}, \ldots, S_{i}, \ldots, S_{n}\right)$ be a honey source of the ABC algorithm. According to the initial processing sequence derived from the join vector and two heuristic rules, the scheduling arrangement in the subsequent stage can be solved. The flow chart of the improved algorithm is shown in Figure 4.

Based on the above analysis, the improved ABC algorithm was used to solve the optimal design model. The honey source $\mathrm{X}$ was defined as the combination of capacity of internal combustion engine $N_{p g u}$, device start-stop coefficient $\alpha$ and electric refrigeration ratio coefficient $\beta: X=\left[N_{p g u}, \alpha, \beta\right]$.

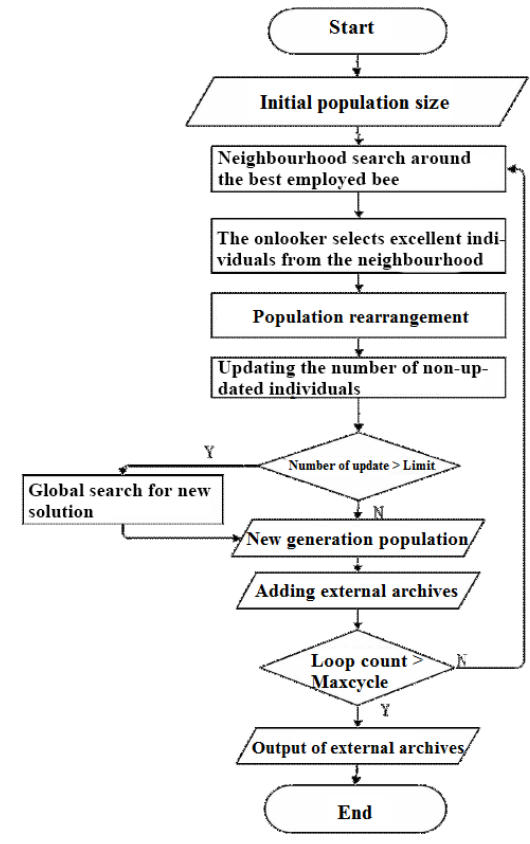

Figure 4. The flow chart of the improved algorithm

\section{SIMULATION TEST AND RESULT ANALYSIS}

A simulation model was constructed in light of the structure of the proposed CCHP system. Then, an aquaculture farm in Beijing was cited as an example to verify our design optimization method. Its annual hourly load data of the farm is shown in Figure 5.

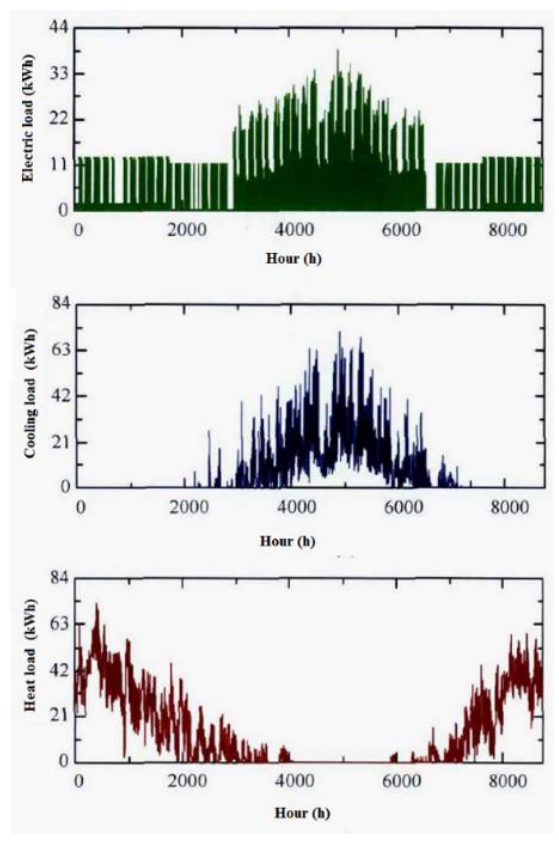

Figure 5. Annual hourly load data of the farm

According to the climate of Beijing, May to September was defined as summer, January to March as winter, and the other months as the transitional season. In summer, the recovered waste heat is mainly used to drive the absorption refrigerator, while the auxiliary electric refrigerator works to realize mixed cooling. In winter and the transitional season, the recovered waste heat is directly heated; if the heating capacity is lacking, 
the gas boiler will make up for the gap. Table 1 lists the parameters of each component in the CCHP system.

Table 1. The parameters of each component in the CCHP system

\begin{tabular}{c|c}
\hline Name & Value \\
\hline Rated COP of absorption refrigerator & 0.51 \\
\hline Rated COP of electric refrigerator & 4.47 \\
\hline Efficiency of gas boiler & 0.77 \\
\hline Efficiency of power grid & 0.32 \\
\hline Efficiency of power transmission & 0.96 \\
\hline Efficiency of heat exchanger & 0.82 \\
\hline
\end{tabular}

The multi-objective $\mathrm{ABC}$ algorithm was adopted to solve the optimization problem. The fitness values of objective function through the convergent process is shown in Figure 6.

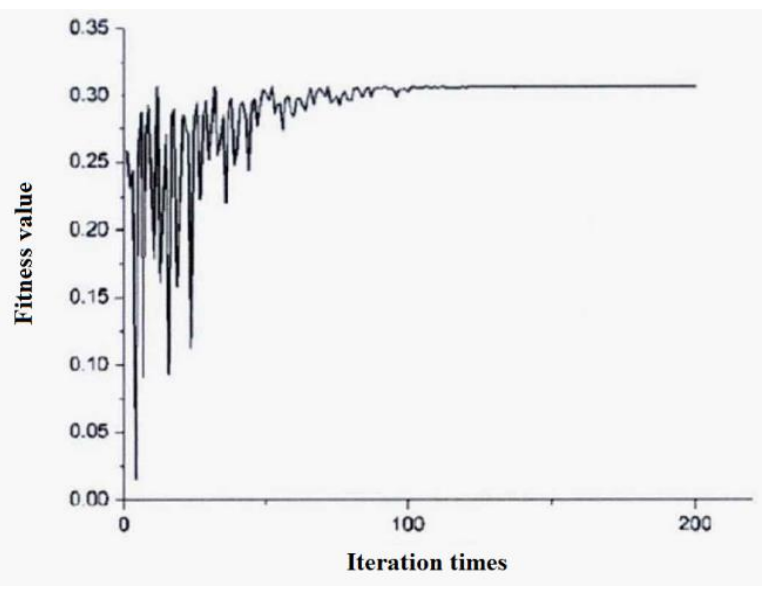

Figure 6. Fitness values of the objective function through the convergent process

As shown in Figure 6, the objective function converged rapidly within 200 iterations, indicating the effectiveness of our multi-objective optimization model.

\section{CONCLUSIONS}

This paper probes deep into the operation features and defects of small-scale CCHP system under the basic operation mode, and then proposes the design optimization method and efficient operation strategy to enhance the system performance in energy efficiency, economy and greenness. Meanwhile, the population coding and selection process of multi-objective artificial bee colony (ABC) algorithm was improved to solve the proposed optimization model. Finally, the proposed optimization model was verified through simulation experiments. The research findings shed new light on the operation features of the CCHP system under the optimization strategy, and the coupling between the different objectives.

\section{REFERENCES}

[1] Jing, Y., Bai, H., Zhang, J. (2012). Multi-objective optimization design and operation strategy analysis of a solar combined cooling heating and power system. Proceedings of the Csee, 32(20): 82-87.
[2] Fang, F., Wang, Q.H., Yang, S. (2012). A novel optimal operational strategy for the CCHP system based on two operating modes. IEEE Transactions on Power Systems, 27(2):

https://doi.org/10.1109/TPWRS.2011.2175490

1032-1041.

[3] Li, G., Wang, R., Zhang, T., Ming, M.J. (2018). Multiobjective optimal design of renewable energy integrated CCHP system using PICEA-g. Energies, 11(4): 743-755. https://doi.org/10.3390/en11040743

[4] Abdollahi, G., Sayyaadi, H. (2013). Application of the multi-objective optimization and risk analysis for the sizing of a residential small-scale CCHP system. Energy and Buildings, 60: 330-344. https://doi.org/10.1016/j.enbuild.2013.01.026

[5] Ahmadi, P., Almasi, A., Shahriyari, M., Dincer, I. (2012). Multi-objective optimization of a combined heat and power (CHP) system for heating purpose in a paper mill using evolutionary algorithm. International Journal of Energy Research, 36(1): 46-63. http://dx.doi.org/10.1002/er.1781

[6] Lorestani, A., Ardehali, M.M. (2018). Optimal integration of renewable energy sources for autonomous tri-generation combined cooling, heating and power system based on evolutionary particle swarm optimization algorithm. Energy, 145: 839-855. https://doi.org/10.1016/j.energy.2017.12.155

[7] Zoolfakar, A., Rani, R., Morfa, A., O’Mullane, A.P., Kalantar-zadeh, K. (2014). Nanostructured copper oxide semiconductors: A perspective on materials, synthesis methods and applications. Journal of Materials Chemistry $\quad$ C, $2(27)$ : $\quad 5247-5270$. https://doi.org/10.1039/C4TC00345D

[8] Fang, F., Wang, Q.H., Shi, Y. (2012). A novel optimal operational strategy for the CCHP system based on two operating modes. IEEE Transactions on Power Systems, 27(2): https://doi.org/10.1109/TPWRS.2011.2175490

[9] Arosio, S., Guilizzoni, M., Pravettoni, F. (2011). A model for micro-trigeneration systems based on linear optimization and the Italian tariff policy. Applied Thermal Engineering, 31(14-15): 2292-2300. https://doi.org/10.1016/j.applthermaleng.2011.03.027

[10] Teng, X., Xin, W., Chen, Y., Shi, W. (2014). A simple method to determine the optimal gas turbine capacity and operating strategy in building cooling, heating and power system. Energy \& Buildings, 80: 623-630. https://doi.org/10.1016/j.enbuild.2014.04.056

[11] Liu, M., Shi, Y., Fang, F. (2012). A new operation strategy for CCHP systems with hybrid chillers. Applied Energy, 95(1): 164-173. https://doi.org/10.1016/j.apenergy.2012.02.035

[12] Zhang, C., Lyu, H.L., Duan, P., Hua, X.H. (2015). Semitensor product based fuzzy modeling for CCHP system. Building Electricity, 34(9): 59-64.

[13] Jing, Y.Y., Bai, H., Wang, J.J. (2012). A fuzzy multicriteria decision-making model for CCHP systems driven by different energy sources. Energy Policy, 42(1): 286-296. https://doi.org/10.1016/j.enpol.2011.11.085

[14] Ali, S., Kim, D.H. (2015). Optimized power control methodology using genetic algorithm. Wireless Personal Communications, $\quad 83(1)$ : 493-505. https://doi.org/10.1007/s11277-015-2405-3

[15] Pantaleo, A.M., Giarola, S., Bauen, A., Shah, N. (2014). Integration of biomass into urban energy systems for heat 
and power. Part I: An MILP based spatial optimization methodology. Energy Conversion and Management, 83: 347-361.

https://doi.org/10.1016/j.enconman.2014.03.050

[16] Jayasekara, S., Halgamuge, S.K., Attalage, R.A., Rajarathne, R. (2014). Optimum sizing and tracking of combined cooling heating and power systems for bulk energy consumers. Applied Energy, 118: 124-134. https://doi.org/10.1016/j.apenergy.2013.12.040

[17] Wang, J.J., Fu, C., Yang, K., Zhang, X.T., Shi, G.H., Zha, J. (2013). Reliability and availability analysis of redundant BCHP (building cooling, heating and power) system. $\quad$ Energy, 61(1): 531-540. https://doi.org/10.1016/j.energy.2013.09.018
[18] Sun, G.X., Bin, S. (2017). Router-level internet topology evolution model based on multi-subnet composited complex network model. Journal of Internet Technology, 18(6):

$1275-1283$ http://dx.doi.org/10.6138/JIT.2017.18.6.20140617

[19] Rohaninejad, M., Kheirkhah, A., Fattahi, P., Nouri, B.V. (2015). A hybrid multi-objective genetic algorithm based on the ELECTRE method for a capacitated flexible job shop scheduling problem. The International Journal of Advanced Manufacturing Technology, 77(1-4): 51-66. https://doi.org/10.1007/s00170-014-6415-1 